\title{
Risk stratification for upper tract urinary carcinoma
}

\author{
Daniel Benamran ${ }^{1,2}$, Thomas Seisen², Elias Naoum², Christophe Vaessen², Jérome Parra ${ }^{2}$, Pierre Mozer², \\ Shahrokh F. Shariat ${ }^{3}$, Morgan Rouprêt ${ }^{2}$
}

${ }^{1}$ Division of Urology, Geneva University Hospitals, Geneva, Switzerland; ${ }^{2}$ Sorbonne Université, GRC n5, ONCOTYPE-URO, AP-HP, Urology Department, Hôpital Pitié-Salpêtrière, F-75013 Paris, France; ${ }^{3}$ Department of Urology, Medical University of Vienna, Vienna, Austria

Contributions: (I) Conception and design: D Benamran, M Roupret; (II) Administrative support: M Roupret; (III) Provision of study material: D Benamran, T Seisen; (IV) Data analysis and interpretation: D Benamran, E Naoum, P Mozer; (V) Data analysis and interpretation: D Benamran, E Naoum, P Mozer; (VI) Manuscript writing: All authors; (VII) Final approval of manuscript: all authors.

Correspondence to: Morgan Rouprêt, MD, PhD. Hôpital Pitié-Salpêtrière, 47-83 Boulevard de l'hôpital, 75013 Paris, France.

Email: morgan.roupret@aphp.fr.

\begin{abstract}
Upper tract urinary carcinoma (UTUC) is a heterogeneous group of rare tumors. The aim of this article is to critically review current therapeutic strategies and to propose a change in the riskstratification of the disease. A non-systematic review of the literature was performed using the Medline database with the search terms: "upper tract urothelial carcinoma" together with "prognostic factor", "risk stratification", "risk factor", "recurrence", "predictive tool”, "nomograms" and "treatment". Preoperative risk factors can be viewed as patient-related risk factors (gender, age, ethnicity, body mass index, smoking status, or genetic factors), or tumor-related risk factors (stage, grade, size, architecture, multifocality, ureteric obstruction). Several biomarkers, available either in blood, urine, or the tumor itself have also been proposed. However, many of these prognostic factors lack accuracy and validation in predicting oncological outcomes, despite their use in predictive tools. After risk stratification, kidney-sparing strategies should be considered (endoscopic management and segmental ureterectomy) and could benefit from new diagnostic tools and technical improvements in in situ adjuvant endocavitary instillations. Radical nephroureterectomy remains the first choice therapy for high-grade disease and will probably be associated with other treatments in the future (lymphadenectomy, perioperative chemotherapy, or immunotherapy). In view of the major recent improvements in UTUC treatment strategies, a new classification should be proposed, including low-, intermediate-, high- and very high-risk disease. Subgroup analysis of good quality trials and better understanding of UTUC risk factors will help validate this new approach toward more personalized medicine.
\end{abstract}

Keywords: Cancer; risk factors; survival; urothelial; stratification

Submitted Oct 04, 2019. Accepted for publication Dec 11, 2019.

doi: $10.21037 /$ tau.2019.12.21

View this article at: http://dx.doi.org/10.21037/tau.2019.12.21

\section{Introduction}

Upper tract urinary carcinoma (UTUC) is a rare type of urothelial cancer, with an estimated annual incidence of 1-2 cases per 100,000 inhabitants (1). It accounts for $5-10 \%$ of all urothelial carcinomas (2), and while it shares similar features with urothelial carcinoma of the bladder, it represents a specific disease with specific management and recommendations.
Radical nephroureterectomy (RNU) is still considered to be the standard of care for most localized UTUCs in various recommendations. However, RNU carries a significant risk of morbidity and impaired renal function. Various strategies have emerged and are constantly developing to manage UTUC conservatively, including endoscopic or segmental resection, sometimes associated with perioperative (neo-) adjuvant treatment, whether localized or systemic. These strategies were initially limited to imperative indications 
(e.g., anatomic or functional solitary kidney), but the development of ureteroscopy over the past few decades has enhanced its role in the management of UTUC (3). These kidney-sparing strategies (KSS) appear to be a reasonable and safe option in well-selected patients even with a normal and functional contralateral kidney. Survival outcomes are similar after KSS or RNU in low-risk patients according to an EAU guideline panel systematic review (4).

As in bladder cancer, patient selection and risk stratification appears to be of utmost importance in UTUC. However, while bladder cancer has many validated peroperative risk stratification tools and objective criteria, UTUC patients remain poorly selected. Due to the low incidence of UTUC, the majority of studies consist of small, single institution, retrospective cohorts resulting in the lack of level 1 evidence for high-grade recommendations.

The aim of this brief review is to provide an overview of current risk stratification tools for UTUC patients and to offer an insight into how these patients can be better stratified in the light of new management options.

\section{Materials and methods}

\section{Literature review}

A non-systematic literature search was performed using the PubMed/Medline databases. Articles published after 2000 were screened using a combination of the following terms: "upper tract urothelial carcinoma" together with "prognostic factor", "risk stratification", "risk factor", "recurrence", "predictive tool", "nomograms" and "treatment". The recommendations of the main urological societies were also screened and additional articles were selected by crossreferencing the bibliography of previously selected articles.

\section{Preoperative predictive factors for UTUC}

\section{Patient-related factors}

Although the incidence of UTUC is higher in men than in women, female gender was thought to be associated with a worse oncologic outcome (5). However, after adjustment for other prognostic factors, no significant difference was found in cancer-specific (CSS) and overall survival (OS) between the two genders $(5,6)$.

The same goes for advanced age. While many epidemiological studies suggested that advanced age was an independent factor for tumor recurrence and shorter CSS $(7,8)$, as well as for invasive tumor patterns, large multi-institutional studies showed that advanced age was not associated with worse survival when adjusted for performance status (9).

The relationship between ethnicity and UTUC outcomes is also debatable. African-Americans were found to have a shorter survival than other ethnic groups (10), but this finding could be explained by worse access to care for African-Americans in the USA. Another study comparing Japanese, European, and US Caucasian patients did not find any differences in survival (11).

Altogether, the differences observed regarding gender, age, or ethnicity may reflect change in tumor biology and aggressiveness, and also heterogeneity in access to health care, they should in our opinion not be considered in treatment decision algorithms.

On the other hand, smoking is a well-established risk factor for UTUC. Long-term smokers (more than 20 cigarettes a day for more than 20 years) are at greater risk of more advanced disease stage or experiencing recurrence after RNU (12). Patients with specific factors for UTUC, such as Lynch syndrome, or long-term exposure to aristolochic acid or phenacetin should also be considered at higher risk of recurrence (13).

\section{Tumor-related factors}

The most accurate independent factors for outcome in UTUC remain stage and grade, which are evaluated by cytology, biopsy of the tumor, and imaging techniques. High-grade cytology is a significant predictor of more advanced disease, non-organ confined disease, loco-regional and distant recurrence (14). Cytology obtained during voiding or cystoscopy suffers from low sensitivity for lowrisk disease (15), but has very good specificity if obtained in situ (selective ureteral cytology), notably for high-grade disease, exceeding $90 \%$ (16). Ureteroscopic biopsy has good accuracy for tumor grade, ranging from 69-91\% (17), but low performance for tumor stage. However, bioptic grading may be used to predict tumor stage: $68-100 \%$ of grade 1 tumors on biopsy are non-muscle invasive tumors, while $62-100 \%$ of grade 3 tumors on biopsy are $\geq \mathrm{p} T 2$ tumors (18). Pre-operative T-staging therefore relies mainly on imaging. The positive predictive value of computed tomography (CT) to detect muscle invasive tumors is high, despite a risk of under-staging tumors. Wang et al. (19) recently demonstrated that tumor stage is a significant prognostic factor for CSS and all-cause mortality in UTUC patients, and tumor stage and grade should therefore be used in decision treatment algorithms. 
Ureteroscopy and imaging also provide important information about localization, size, and multifocality of the tumor. The impact of tumor location on oncologic outcomes is still under debate. Some studies have suggested that the rate of organ-confined tumors is the same irrespective of whether the tumor lies in the ureter or the renal pelvis (20), while others show that ureteral location is associated with a worse outcome (21). Tumors $>3 \mathrm{~cm}$ in size were associated with a worse outcome in patients treated by RNU in retrospective studies (22). Similarly, tumor multifocality has been shown to affect CSS after RNU (23), and some authors have suggested that RNU is a more reasonable treatment option for patients with multifocal disease (24). This also accounts for the difficulty of selective treatment (endoscopic or extirpative) when the tumor is very large or multifocal.

Ureteroscopy can also help identify tumor architecture. Sessile architecture has been shown to be associated with a worse oncologic outcome (non-organ confined disease, high-grade disease, cancer recurrence) than papillary architecture (25). Most studies show that preoperative hydronephrosis is also associated with more advanced disease [more advanced $\mathrm{T}$ stage, more frequent lymphovascular invasion, and high-grade disease (26-28)].

\section{Biomarkers}

Aside from pathologic and radiologic factors like stage, grade, size, and multifocality, genetic markers can help predict the prognosis of UTUC. For example, microsatellite instability has been identified in many cancers, especially those associated with Lynch syndrome, and is associated with a better outcome in T2-T3N0 UTUC patients (29). Low methylation of the VIM promoter predicts worse CSS in UTUC patients (30), while FGFR3 mutations are frequently associated with lower stage and better survival of UTUC patients (31).

Several tissues biomarkers have also been investigated. Ki-67 expression was shown to be associated with adverse pathologic features in 475 patients treated by RNU (32). Similar findings were found for PI3K and Cyclin D (two mTOR biomarkers) (33). PD1 and PD-L1 expression have also been proposed as prognostic factors in UTUC $(34,35)$. In a retrospective cohort of more than 400 patients undergoing extirpative surgery, PD-1 expression was associated with worse CSS and OS. In contrast, PD-L1 expression was predictive of a more favorable outcome.
Blood and urine biomarkers have also been investigated as prognostic markers in UTUC. Pre-operative C-reactive protein level was shown to be associated with a poor prognosis (36). Pre-operative neutrophil: lymphocyte ratio was also significantly associated with worse pathologic features and higher CSS (37). Other easily available markers such as platelet count, fibrinogen, albumin, or hemoglobin have been investigated. Most of these biomarkers however, whether derived from the tumor itself, from blood, or from urine, still lack validation in large studies.

\section{Predictive tools}

Based on these identified prognostic factors, and despite the challenge of their accurate characterization based on routine examination through imaging, endoscopy, or biopsy, multiinstitutional research groups have developed per-operative models to predict high-grade, muscle-invasive or non-organ confined disease.

Several models focus on the prediction of muscle-invasive or non-organ confined disease. In 2010, Brien et al. first proposed the combination of hydronephrosis, high-grade disease on biopsy, and positive urinary cytology to predict advanced UTUC in a small retrospective cohort (38). If no criteria were present, the negative predictive value for muscle-invasive or non-organ confined disease reached $100 \%$. However, when all three criteria were met, the positive predictive value for muscle-invasive or non-organ confined disease was $89 \%$ and $73 \%$, respectively. Margulis et al. also proposed a model to predict non-organ confined disease with an accuracy of $77 \%$, associating tumor grade, architecture, and location (39). In 2012, Favaretto et al. combined preoperative hydronephrosis, tumor grade, and location to predict muscle-invasive disease or locally advanced disease with an accuracy of $70 \%$ (40). A cohort of 683 Chinese patients helped to develop a model predicting non-organ confined disease with an accuracy of $79 \%$ based on gender, tumor grade, architecture, and multifocality (41). Finally, another model to predict muscle-invasive or nonorgan confined disease was developed in 2015, including gender, locally advanced stage on pre-operative workup, and positive cytology with an accuracy of $65.3 \%$ and $67.2 \%$ for muscle-invasive and non-organ confined disease respectively (42). These models should be used with care in clinical practice and decision making as none have benefited from external validation. 


\section{Treatment options for localized disease}

\section{KSS}

\section{Endoscopic management and segmental resection}

Ureteroscopic management of UTUC has emerged as an alternative to RNU for localized disease. In a single center, retrospective cohort with 52 months follow-up, 112 patients with tumors for which complete endoscopic eradication was achievable were treated with flexible ureteroscopic laser photoablation (43); the progressionfree survival rate was $70 \%$. The only predictive factor for disease progression was the presence of high-grade tumor ( $75 \%$ vs. $52 \%$ after 52 months). Tumor size $(\leq 1 v s .>1 \mathrm{~cm})$ or multifocality were not independent prognostic factors. Several other authors have reported encouraging results for endoscopic management of UTUC compared to RNU (44) and a meta-analysis comparing endoscopic treatment and RNU demonstrated equivalence between these treatment strategies in selected favorable cases (45). Usually, a biopsy is performed during an endoscopic procedure and treatment is initiated immediately (laser vaporization) as a second step of the procedure regardless of biopsy findings. Thus, one of the key issues of ureteroscopic management is that the tumor is treated based on a subjective decision (size, aspect, multifocality, and the endourologist's own experience) without knowledge of the tumor grade. The use of endoscopic confocal laser endomicroscopy could help identify which are the best candidates for this strategy. Confocal laser endomicroscopy $\left(\right.$ Cellvizio $^{\circledR}$ ) provides microscopic images of tissues in vivo using a low-energy laser light source and can be used in real-time during ureteroscopy. In a small series, Breda et al. (46) were able to identify the correct grading using confocal laser endomicroscopy during endoscopic ablation of UTUC in $13 / 14$ patients. This could allow real-time characterization of the lesion to identify patients with low-grade tumors suitable for conservative management and could be considered as "endoscopic frozen section analysis".

Segmental ureterectomy describes the removal of the part of the ureter where the UTUC is localized, by either ureteroureterostomy or ureteroneocystostomy and allows for concomitant lymphadenectomy. Retrospective studies suggest that segmental ureterectomy has comparable longterm outcomes to RNU (47), with 5-year CSS around $85 \%$ when indicated carefully (4). Laparoscopic and robotassisted ureterectomy have helped to decrease per-operative morbidity. A double approach with intra-operative ureteroscopy can also help to identify the limits of the tumor while performing robotic ureterectomy. A good tip is the use of near-infrared light mode (FireFly mode), which can be activated without any intravenous indocyanine green injection to better identify the ureteroscope and the tumor margins (see Figure 1). Intravenous indocyanine green injection could also help to enhance local visualization of ureteral vascularization.

\section{In situ endocavitary instillations}

There is a strong rationale for endocavitary instillation of topical (neo-)adjuvant treatments, either antegradely or retrogradely. The outcomes associated with adjuvant endocavitary instillations have been poorly assessed in the literature (44). Like urothelial carcinoma of the bladder, the topical agents used are commonly mitomycin $\mathrm{C}$ and BCG. The major drawback is the reduced exposure time of these topical agents to the tumor site. To overcome this drawback, a novel hydrogel polymer with reverse thermal gelation properties (liquid at cold temperatures and solid at body temperature) might promote high-dose delivery of mitomycin $\mathrm{C}$ into the upper urinary tract. Several preclinical studies have already been published and an ongoing phase 3 trial (Olympus Study) (48) is underway to assess the safety and efficacy of such instillations in patients with lowgrade UTUC.

\section{Radical surgery}

RNU with bladder-cuff excision is the standard treatment for high-grade UTUC (2). Bladder-cuff excision is mandatory due to the high recurrence rate in the ureteral stump (16-58\%). Like segmental resections, RNU can be done through a minimally invasive approach. Laparoscopy can help diminish the postoperative burden and has been shown to be non-inferior to the open approach (49). The value of lymphadenectomy during RNU is still unclear but allows better staging to help post-operative adjuvant treatment. The anatomic landmarks and extent depend on the tumor localization and no strong recommendations can currently be made. A single post-operative intravesical mitomycin instillation should always be considered since its effect on reducing intravesical recurrence after RNU has been demonstrated (50).

\section{Systemic treatment}

It is very likely that systemic chemotherapy and immunotherapy will be part of the therapeutic armamentarium for very high-risk localized disease in the future, even for non-metastatic UTUC patients. The most interesting results presented recently are those from POUT Trial, a phase III randomized study conducted in the UK since 2012 (51). A total of 261 patients who underwent 


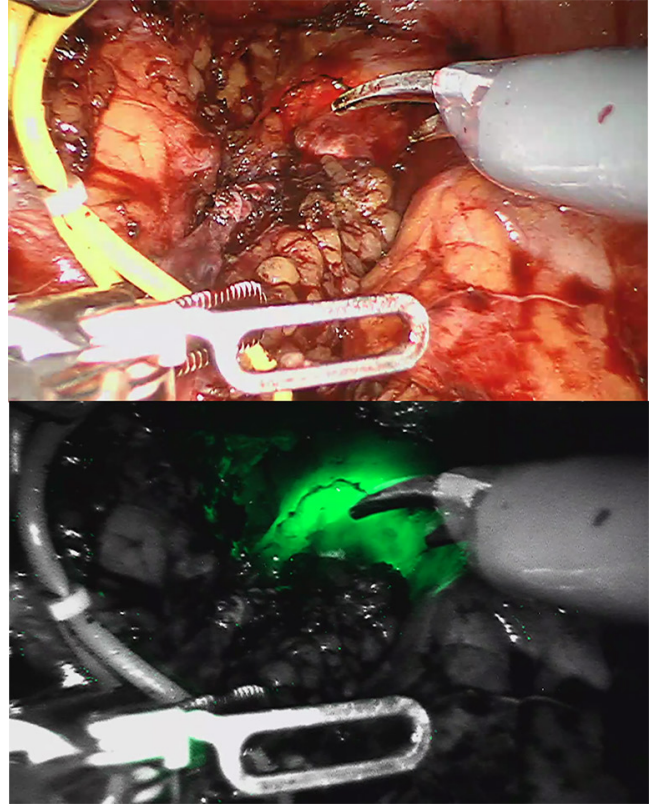

Figure 1 Intraoperative view during segmental resection in regular light mode (top image) and near-infrared light (Firefly ${ }^{\circledR}$ ) mode (bottom image). The ureteroscope is inserted up to the margin of the ureteral tumor. When the Firefly ${ }^{\circledR}$ mode is activated, the light of the ureteroscope is easily visible in green.

RNU for pT2-T4N0-3 M0 UTUC were randomized to four cycles of adjuvant chemotherapy or observation. Most of the patients were pT3 (approximately 65\%) and pN0 $(91 \%)$. The authors found a significant difference in diseasefree survival at 2 years in favor of chemotherapy (51\% vs. $70 \%)$, despite a high rate of grade $\geq 3$ toxicity (62\%). The trial was terminated early because of efficacy favoring the chemotherapy arm and paved the way for the development of a new trial (POUT2) that will randomize chemotherapy $v s$. a combination of chemotherapy and immunotherapy in the perioperative setting.

\section{Can we improve the current risk stratification strategy?}

The treatment strategy for localized disease without evidence of metastasis should be based on careful risk stratification. According to the most recent EAU guidelines, as tumor stage is difficult to identify clinically in UTUC, it is useful to "risk stratify" UTUC between low- and highrisk tumors to identify those patients who are more suitable for KSS rather than radical extirpative surgery. KSS should always be considered in cases of absolute indication, but also for localized disease amenable to complete excision (42). Patients should also be informed about the risk of more aggressive treatment, recurrence, or progression, and accept strict surveillance protocols. KSS for low-risk UTUC reduces the morbidity associated with radical surgery, without compromising oncologic outcomes or kidney function, as stated in a systematic review from the EAU guidelines panel. In low-risk cancers, it is the preferred approach with survival being similar after KSS vs. RNU. This option should therefore be discussed in all low-risk cases, irrespective of the status of the contralateral kidney.

International guidelines recommend risk stratification for many urologic cancers, such as prostate cancer or nonmuscle invasive bladder cancer. The concept of low-risk and high-risk tumors has been developed by urological societies such as the European Association of Urology (EAU) (2) or the International Consultation on Urological Disease (ICUD) $(15,52)$. A low-risk tumor is defined as unifocal disease, low-grade status on cytology or biopsy, and no invasive aspect on imaging. Historically, the maximum tumor size for low-risk disease was $1 \mathrm{~cm}$, even though no data supported a poorer prognosis for tumors $>1 \mathrm{~cm}$; this was due to technical difficulties in conservatively managing tumors $>1 \mathrm{~cm}$. However, this limit has now been changed to $2 \mathrm{~cm}$, since larger tumors should not be an exclusion criterion for endoscopic management or segmental resection. High-risk tumors include any high-grade status (on cytology or biopsy), lesions $>2 \mathrm{~cm}$, presenting with hydronephrosis or multifocality. A history of radical cystectomy or variant histology are also criteria for highrisk tumors.

In our opinion, elective indications for endoscopic management or segmental resection should be reserved for localized low-grade disease amenable to complete resection. Multifocality or large papillary tumors should not be excluded from elective indications.

The current criteria used to stratify patients remain limited. Molecular characterization will probably help to improve risk stratification in the future, but most molecular characterization studies have focused on RNU specimens and their predictive value on biopsies needs validation. Furthermore, most studies suffer from their retrospective design and small sample size. Therefore, further collaborative efforts are needed to validate predictive models and to identify and validate new biomarkers from endoscopic biopsies. Without these efforts, recommendations on the grade for risk stratification will remain poor. In the meantime, we strongly believe 
Table 1 Risk stratification according to the European Association of Urology

Low-risk upper tract urinary carcinoma (UTUC) (all criteria must
be present)
Unifocal disease
Tumour size $<2 \mathrm{~cm}$
Low-grade cytology
Low-grade biopsy
No invasive aspect on CT-urography
High-risk UTUC (any criteria may be present)
Multifocal disease
Tumour size $>2$ cm
High-grade cytology
High-grade biopsy
Hydronephrosis
Previous radical cystectomy for bladder cancer
Variant histology

Table 2 Proposed new improved stratification in four groups

Low-risk upper tract urinary carcinoma (UTUC)
Unifocal disease
Low-grade cytology
No invasive aspect on CT-urography
Intermediate-risk UTUC
Multifocal disease if endoscopic or segmental treatment
possible
Low-grade cytology
No invasive aspect on CT-urography
High-risk UTUC
High-grade cytology or biopsy
Carcinoma in situ or variant histology
Sessile lesions
Invasive aspect on CT-urography
Large, bulky disease
Very high-risk subgroup
Locally advanced or non-organ confined disease

that risk stratification needs improvement in the light of new treatment options. The current EAU classification proposal considers only two categories (Table 1) (2). This is not enough in the light of the treatment options currently available or under development. For example, low-risk tumors should certainly be treated endoscopically, usually when the disease is a small low-grade unifocal papillary tumor without any significant risk factor. An intermediaterisk group could be low-grade tumors of higher volume, which would still be accessible for endoscopic treatment or segmental resection, and would possibly benefit from in situ adjuvant instillation to prevent local recurrence. High-grade tumors or invasive disease should remain classified as high-risk disease requiring RNU. In our opinion, lymphadenectomy should be performed when possible, since its prognostic and therapeutic value has been suggested, mostly for muscle-invasive or locally advanced disease (53). A subgroup of these high-risk patients might be cured by surgery only, while another subgroup of very high-risk patients will benefit from perioperative systemic treatment. Subgroup analyses of ongoing trials will certainly help to better stratify the high-risk patients who could avoid the morbidity of systemic perioperative treatments in the same way that low-risk or intermediate-risk patients should avoid the morbidity of RNU. Table 2 summarizes this new proposal and could be the basis for a new risk-adapted approach depending on the localization of the tumor as suggested in Figure 2.

This new classification has not yet been validated and certain treatment options are optional. However, the timeline of evidence-based medicine is not always similar to the timeline of clinical daily practice. With all new possibilities on the table, we are convinced that some colleagues are already thinking out of the box and are moving forward in the direction of personalized medicine for UTUC management.

\section{Conclusions}

Current international guidelines recommend a risk-adapted approach to UTUC management. New tools and treatment options are being developed to help stratify our patients better and to offer them more options. A new stratification is therefore needed to integrate them when they have been fully validated. 


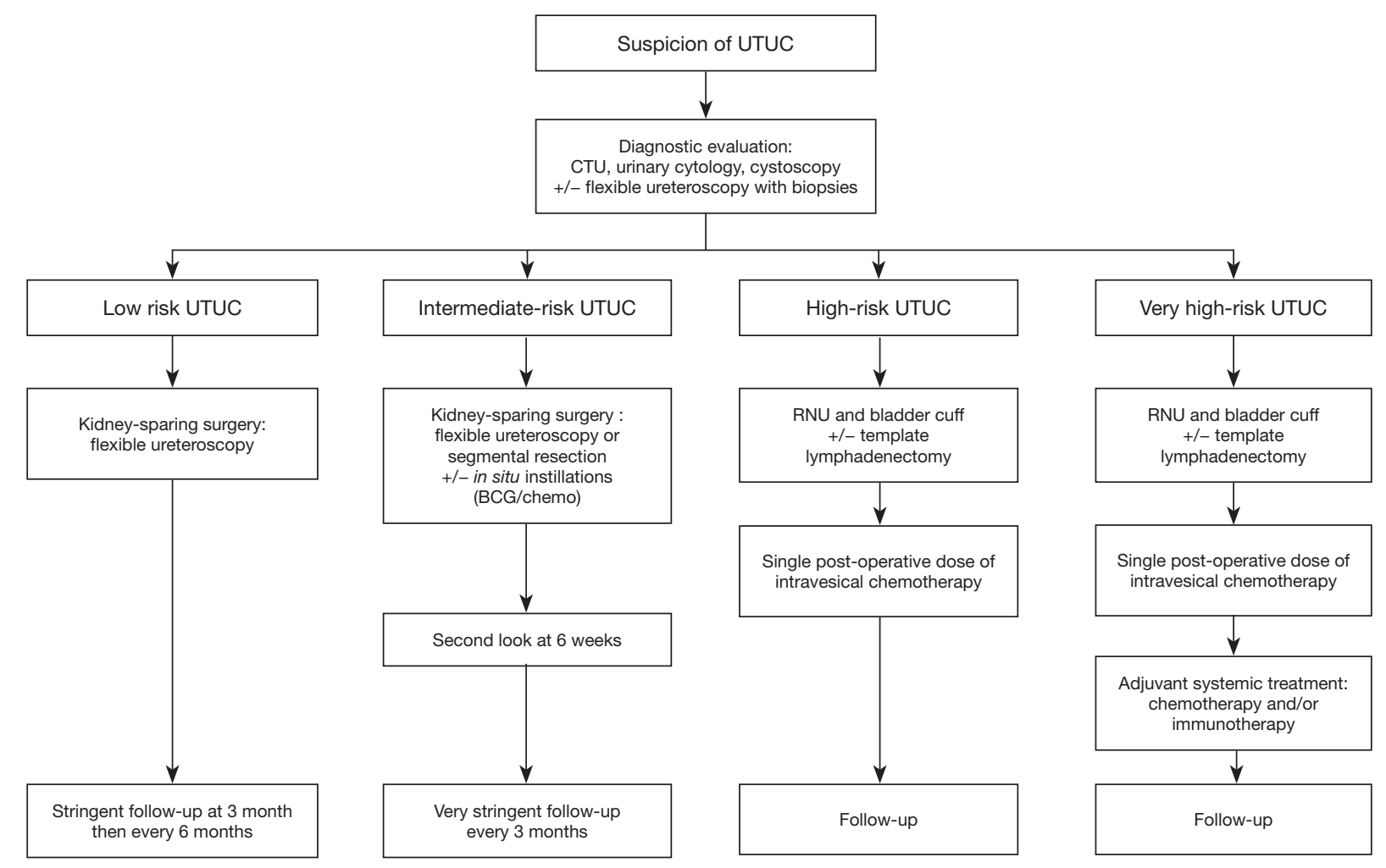

Figure 2 Proposed new risk-adapted approach.

\section{Acknowledgments}

Funding: None.

\section{Footnote}

Provenance and Peer Review: This article was commissioned by the Guest Editors (John J. Knoedler and Jay D. Raman) for the series "Upper-Tract Urothelial Carcinoma: Current State and Future Directions" published in Translational Andrology and Urology. The article was sent for external peer review organized by the Guest Editors and the editorial office.

Conflicts of Interest: All authors have completed the ICMJE uniform disclosure form (available at http:// dx.doi.org/10.21037/tau.2019.12.21). The series "UpperTract Urothelial Carcinoma: Current State and Future Directions" was commissioned by the editorial office without any funding or sponsorship. The authors have no other conflicts of interest to declare.
Ethical Statement: The authors are accountable for all aspects of the work in ensuring that questions related to the accuracy or integrity of any part of the work are appropriately investigated and resolved.

Open Access Statement: This is an Open Access article distributed in accordance with the Creative Commons Attribution-NonCommercial-NoDerivs 4.0 International License (CC BY-NC-ND 4.0), which permits the noncommercial replication and distribution of the article with the strict proviso that no changes or edits are made and the original work is properly cited (including links to both the formal publication through the relevant DOI and the license). See: https://creativecommons.org/licenses/by-nc-nd/4.0/.

\section{References}

1. Siegel RL, Miller KD, Jemal A. Cancer statistics 2016. CA Cancer J Clin 2016;66:7-30.

2. Rouprêt M, Babjuk M, Compérat E, et al. European Association of Urology guidelines on upper urinary 
tract urothelial carcinoma: 2017 Update. Eur Urol 2018;73:111-22.

3. Audenet F, Traxer O, Yates DR, et al. Potential role of photodynamic techniques combined with new generation flexible ureterorenoscopes and molecular markers for the management of urothelial carcinoma of the upper urinary tract. BJU Int 2012;109:608-13.

4. Seisen T, Peyronnet B, Dominguez-Escrig JL, et al. Oncologic outcomes of kidney-sparing surgery versus radical nephroureterectomy for upper tract urothelial carcinoma: a systematic review by the EAU non-muscle invasive bladder cancer guidelines panel. Eur Urol 2016;70:1052-68.

5. Lughezzani G, Sun M, Perrotte P, et al. Gender-related differences in patients with stage I to III upper tract urothelial carcinoma: results from the surveillance, epidemiology, and end results database. Urology 2010;75:321-7.

6. Shariat SF, Favaretto RL, Gupta A, et al. Gender differences in radical nephroureterectomy for upper tract urothelial carcinoma. World J Urol 2011;29:481-6.

7. Shariat SF, Godoy G, Lotan Y, et al. Advanced patient age is associated with inferior cancer-specific survival after radical nephroureterectomy. BJU Int 2010;105:1672-7.

8. Margulis V, Shariat SF, Matin SF, et al. Outcomes of radical nephroureterectomy: a series from the Upper Tract Urothelial Carcinoma Collaboration. Cancer 2009;115:1224-33.

9. Martinez-Salamanca JI, Shariat SF, Rodriguez JC, et al. Prognostic role of ECOG performance status in patients with urothelial carcinoma of the upper urinary tract: an international study. BJU Int 2012;109:1155-61.

10. Raman JD, Messer J, Sielatycki JA, et al. Incidence and survival of patients with carcinoma of the ureter and renal pelvis in the USA, 1973-2005. BJU Int 2011;107:1059-64.

11. Matsumoto K, Novara G, Gupta A, et al. Racial differences in the outcome of patients with urothelial carcinoma of the upper urinary tract: an international study. BJU Int 2011;108:E304-9.

12. Rink M, Xylinas E, Margulis V, et al. Impact of smoking on oncologic outcomes of upper tract urothelial carcinoma after radical nephroureterectomy. Eur Urol 2013;63:1082-90.

13. Ouzzane A, Rouprêt M, Leon P, et al. Epidemiology and risk factors of upper urinary tract tumors: Literature review for the yearly scientific report of the French National Association of Urology. Prog Urol 2014;24:966-76.
14. Mbeutcha A, Rouprêt M, Kamat AM, et al. Prognostic factors and predictive tools for upper tract urothelial carcinoma: a systematic review. World J Urol 2017;35:337-53.

15. Mandalapu RS, Remzi M, de Reijke TM, et al. Update of the ICUD-SIU consultation on upper tract urothelial carcinoma 2016: treatment of low-risk upper tract urothelial carcinoma. World J Urol 2017;35:355-65.

16. Chen L, He H, Zarka MA, et al. Upper tract urinary cytology to detect upper tract urothelial carcinoma: Using the Johns Hopkins Hospital template and evaluation of its feasibility. Cytojournal 2015;12:17.

17. Khene ZE, Mathieu R, Kammerer-Jacquet SF, et al. Risk stratification for kidney sparing procedure in upper tract urothelial carcinoma. Transl Androl Urol 2016;5:711-9.

18. Cutress ML, Stewart GD, Zakikhani P, et al. Ureteroscopic and percutaneous management of upper tract urothelial carcinoma (UTUC): systematic review. BJU Int 2012;110:614-28.

19. Wang LJ, Chou WC, Pang ST, et al. Risk stratification of upper urinary tract urothelial carcinoma patients for survival prediction: a simple summation scoring method. J Cancer 2018;9:2284-94.

20. Wu Y, Dong Q, Liu L, et al. The impact of tumor location and multifocality on prognosis for patients with upper tract urothelial carcinoma: a meta-analysis. Sci Rep 2014;4:6361.

21. Yafi FA, Novara G, Shariat SF, et al. Impact of tumour location versus multifocality in patients with upper tract urothelial carcinoma treated with nephroureterectomy and bladder cuff excision: a homogeneous series without perioperative chemotherapy. BJU Int 2012;110:E7-13.

22. Simone G, Papalia R, Loreto A, et al. Independent prognostic value of tumour diameter and tumour necrosis in upper urinary tract urothelial carcinoma. BJU Int 2009;103:1052-7.

23. Chromecki TF, Cha EK, Fajkovic H, et al. The impact of tumor multifocality on outcomes in patients treated with radical nephroureterectomy. Eur Urol 2012;61:245-53.

24. Baard J, de Bruin DM, Zondervan PJ, et al. Diagnostic dilemmas in patients with upper tract urothelial carcinoma. Nat Rev Urol 2017;14:181-91.

25. Fan B, Hu B, Yuan Q, et al. Impact of tumor architecture on disease recurrence and cancer-specific mortality of upper tract urothelial carcinoma treated with radical nephroureterectomy. Tumour Biol 2017;39:1010428317710822. 
26. Ito Y, Kikuchi E, Tanaka N, et al. Preoperative hydronephrosis grade independently predicts worse pathological outcomes in patients undergoing nephroureterectomy for upper tract urothelial carcinoma. J Urol 2011;185:1621-6.

27. Ng CK, Shariat SF, Lucas SM, et al. Does the presence of hydronephrosis on preoperative axial CT imaging predict worse outcomes for patients undergoing nephroureterectomy for upper-tract urothelial carcinoma? Urol Oncol 2011;29:27-32.

28. Messer JC, Terrell JD, Herman MP, et al. Multiinstitutional validation of the ability of preoperative hydronephrosis to predict advanced pathologic tumor stage in upper-tract urothelial carcinoma. Urol Oncol 2013;31:904-8.

29. Rouprêt M, Fromont G, Azzouzi AR, et al. Microsatellite instability as predictor of survival in patients with invasive upper urinary tract transitional cell carcinoma. Urology 2005;65:1233-7.

30. Monteiro-Reis S, Leça L, Almeida M, et al. Accurate detection of upper tract urothelial carcinoma in tissue and urine by means of quantitative GDF15, TMEFF2 and VIM promoter methylation. Eur J Cancer 2014;50:226-33.

31. van Oers JMM, Zwarthoff EC, Rehman I, et al. FGFR3 mutations indicate better survival in invasive upper urinary tract and bladder tumours. Eur Urol 2009;55:650-7.

32. Krabbe L-M, Bagrodia A, Haddad AQ, et al. Multiinstitutional validation of the predictive value of $\mathrm{Ki}-67$ in patients with high grade urothelial carcinoma of the upper urinary tract. J Urol 2015;193:1486-93.

33. Bagrodia A, Krabbe L-M, Gayed BA, et al. Evaluation of the prognostic significance of altered mammalian target of rapamycin pathway biomarkers in upper tract urothelial carcinoma. Urology 2014;84:1134-40.

34. Krabbe LM, Heitplatz B, Preuss S, et al. Prognostic value of PD-1 and PD-L1 expression in patients with high grade upper tract urothelial carcinoma. J Urol 2017;198:1253-62.

35. Miyama Y, Morikawa T, Miyakawa J, et al. The prognostic value of PD-L1 expression in upper tract urothelial carcinoma varies according to platelet count. Cancer Med 2018;7:4330-8.

36. Luo Y, Fu SJ, She DL, et al. Preoperative C-reactive protein as a prognostic predictor for upper tract urothelial carcinoma: A systematic review and meta-analysis. Mol Clin Oncol 2015;3:924-8.
37. Tanaka N, Kikuchi E, Shirotake S, et al. The predictive value of C-reactive protein for prognosis in patients with upper tract urothelial carcinoma treated with radical nephroureterectomy: a multi-institutional study. Eur Urol 2014;65:227-34.

38. Brien JC, Shariat SF, Herman MP, et al. Preoperative hydronephrosis, ureteroscopic biopsy grade and urinary cytology can improve prediction of advanced upper tract urothelial carcinoma. J Urol 2010;184:69-73.

39. Margulis V, Youssef RF, Karakiewicz PI, et al. Preoperative multivariable prognostic model for prediction of nonorgan confined urothelial carcinoma of the upper urinary tract. J Urol 2010;184:453-8.

40. Favaretto RL, Shariat SF, Savage C, et al. Combining imaging and ureteroscopy variables in a preoperative multivariable model for prediction of muscle-invasive and non-organ confined disease in patients with upper tract urothelial carcinoma. BJU Int 2012;109:77-82 .

41. Chen XP, Xiong GY, Li XS, et al. Predictive factors for worse pathological outcomes of upper tract urothelial carcinoma: experience from a nationwide high-volume centre in China. BJU Int 2013;112:917-24.

42. Hurel S, Rouprêt M, Seisen T, et al. Influence of preoperative factors on the oncologic outcome for upper urinary tract urothelial carcinoma after radical nephroureterectomy. World J Urol 2015;33:335-41.

43. Villa L, Haddad M, Capitanio U, et al. Which patients with upper tract urothelial carcinoma can be safely treated with flexible ureteroscopy with holmium: YAG laser photoablation? Long-term results from a high volume. J Urol 2018;199:66-73.

44. Seisen T, Colin P, Rouprêt M. Risk-adapted strategy for the kidney-sparing management of upper tract tumours. Nat Rev Urol 2015;12:155-66.

45. Yakoubi R, Colin P, Seisen T, et al. Radical nephroureterectomy versus endoscopic procedures for the treatment of localised upper tract urothelial carcinoma: A meta-analysis and a systematic review of current evidence from comparative studies. Eur J Surg Oncol 2014;40:1629-34.

46. Breda A, Territo A, Guttilla A, et al. Correlation between confocal laser endomicroscopy (Cellvizio®) and histological grading of upper tract urothelial carcinoma: a step forward for a better selection of patients suitable for conservative management. Eur Urol Focus 2018;4:954-9.

47. Simonato A, Varca V, Gregori A, et al. Elective segmental ureterectomy for transitional cell carcinoma of the ureter: 
long-term follow-up in a series of 73 patients. BJU Int 2012;110:E744-9.

48. Kleinmann N, Pierorazio P, Matin S, et al. Non-surgical managment of low grade upper tract urothelial cancer: an interim analysis of the international multicenter Olympus Trial (NCT02793128). J Urol 2018;199:e1166.

49. Simone G, Papalia R, Guaglianone S, et al. Laparoscopic versus open nephroureterectomy: perioperative and oncologic outcomes from a randomised prospective study. Eur Urol 2009;56:520-6.

50. O'Brien T, Ray E, Singh R, et al. Prevention of bladder tumours after nephroureterectomy for primary upper urinary tract urothelial carcinoma: a prospective, multicentre, randomised clinical trial of a single postoperative intravesical dose of mitomycin $\mathrm{C}$ (the

Cite this article as: Benamran D, Seisen T, Naoum E, Vaessen C, Parra J, Mozer P, Shariat SF, Rouprêt M. Risk stratification for upper tract urinary carcinoma. Transl Androl Urol 2020;9(4):1799-1808. doi: 10.21037/tau.2019.12.21
ODMIT-C Trial). Eur Urol 2011;60:703-10.

51. Birtle AJ, Lewis R, Johnson M, et al. Time to define an international standard of postoperative care for resected upper urinary tract transitional cell carcinoma (TCC) - opening of the peri-operative chemotherapy versus surveillance in upper tract urothelial cancer (POUT) trial. BJU Int 2012;110:919-21.

52. Gakis G, Schubert T, Alemozaffar M, et al. Update of the ICUD-SIU consultation on upper tract urothelial carcinoma 2016: treatment of localized high-risk disease. World J Urol 2017;35:327-35.

53. Seisen T, Shariat SF, Cussenot O, et al. Contemporary role of lymph node dissection at the time of radical nephroureterectomy for upper tract urothelial carcinoma. World J Urol 2017;35:535-48. 